\title{
MicroRNA miR-425 is a negative regulator of atrial natriuretic peptide
}

\author{
Pankaj Arora ${ }^{1,2,3,4}$, Connie Wu ${ }^{5}$, Donald B Bloch ${ }^{6,7}$, Brandi N Davis-Dusenbury ${ }^{8}$, Ester Spagnolli ${ }^{5}$, Akiko Hata9 ${ }^{9}$ \\ Sara Vandenwijngaert ${ }^{10}$, Melissa Swinnen ${ }^{10}$, Stefan Janssens ${ }^{10}$, Emmanuel S Buys ${ }^{5}$, Kenneth D Bloch ${ }^{1,2,5^{*}}$, \\ Christopher Newton-Cheh" ${ }^{1,2,3,4+}$, Thomas J Wang ${ }^{1,2+}$
}

From 6th International Conference on cGMP: Generators, Effectors and Therapeutic Implications Erfurt, Germany. 28-30 June 2013

\section{Background}

Numerous common genetic variants have been linked to blood pressure, but no underlying mechanism has been elucidated. Population studies have revealed that a genetic variant, rs5068 (A/G), is associated with blood pressure and the risk of hypertension. rs5068 lies in the 3' untranslated region (3'UTR) of NPPA, the gene encoding atrial natriuretic peptide (ANP), and presence of the minor G allele is associated with increased circulating ANP levels and reduced blood pressure.

\section{Results}

We hypothesized the existence of a microRNA (miR) that targets the NPPA 3'UTR and that the binding of the miR to the NPPA 3'UTR would be disrupted in transcripts from the rs5068 minor allele. We identified a microRNA, miR-425, that is predicted to bind the sequence spanning rs5068 for the A, but not the G, allele. miR-425 is expressed in human atria and ventricles. Using luciferase3'UTR reporter constructs, we observed that miR-425 could silence reporter mRNAs carrying the NPPA major allele 3'UTR, but not those carrying the minor allele 3'UTR. Similarly, an anti-miR directed against miR-425 augmented expression of the luciferase-NPPA 3'UTR construct containing the major allele but not the minor allele. miR-425 reduced NPPA mRNA levels and ANP synthesis in human cardiomyocytes derived from induced pluripotent stem cells.

\section{Conclusion}

Our studies provide mechanistic insights into how a common genetic variant identified in population genetic studies can regulate ANP levels and blood pressure. miR-425 is a novel regulator of ANP production, raising the possibility that miR-425 antagonists could be used to treat disorders of salt overload, including hypertension and heart failure.

\begin{abstract}
Authors' details
${ }^{1}$ Cardiology Division, Massachusetts General Hospital, Boston, Massachusetts, USA. ${ }^{2}$ Cardiovascular Research Center, Massachusetts General Hospital, Boston, Massachusetts, USA. ${ }^{3}$ Center for Human Genetic Research, Massachusetts General Hospital, Boston, Massachusetts, USA. ${ }^{4}$ Program in Medical and Population Genetics, Broad Institute, Cambridge, Massachusetts, USA. ${ }^{5}$ Department of Anesthesia, Critical Care, and Pain Medicine, Massachusetts General Hospital, Boston, Massachusetts, USA. ${ }^{6}$ Division of Rheumatology, Allergy, and Clinical Immunology, Massachusetts General Hospital, Boston, Massachusetts, USA. ${ }^{7}$ Center for Immunology and Inflammatory Diseases, Massachusetts General Hospital, Boston, Massachusetts, USA. ${ }^{8}$ Stem Cell and Regenerative Biology, Harvard University, Cambridge, Massachusetts, USA. ${ }^{9}$ Cardiovascular Research Institute, University of California, San Francisco, CA, USA. ${ }^{10}$ Department of Cardiovascular Sciences, Gasthuisberg University Hospital, University of Leuven, Belgium.
\end{abstract}

Published: 29 August 2013

doi:10.1186/2050-6511-14-S1-010

Cite this article as: Arora et al: MicroRNA miR-425 is a negative regulator of atrial natriuretic peptide. BMC Pharmacology and Toxicology 2013 14(Suppl 1):010.

\footnotetext{
* Correspondence: kdbloch@partners.org

+ Contributed equally

'Cardiology Division, Massachusetts General Hospital, Boston, Massachusetts, USA

Full list of author information is available at the end of the article
}

(c) 2013 Arora et al; licensee BioMed Central Ltd. This is an Open Access article distributed under the terms of the Creative Commons 